\title{
Measurements of transmural strain variations by two dimensional ultrasound speckle tracking
}

\author{
Lysiansky Michael ${ }^{1}$, Bachner-Hinenzon Noa ${ }^{1}$, Khamis Hanan $^{1}$, Smirin Nahum ${ }^{1}$, Lysyansky Peter ${ }^{2}$, \\ Friedman $\mathrm{Zvi}^{2}$, Shimoni Sara ${ }^{3}$, Fehske Wolfgang ${ }^{4}$, Adam Dan ${ }^{1}$ \\ 1. Faculty of Biomedical Engineering, Technion-IIT, Technion City, ISRAEL. 2. General Electric Healthcare, Israel. 3. \\ Department of cardiology, Kaplan medical center, Rehovot, Israel. 4. Klinik für Innere Medizin und Kardiologie, St. Vinzenz \\ Hospital, Cologne, Germany.
}

Correspondence: Bachner-Hinenzon Noa. Address: Faculty of Biomedical engineering, Technion city, Israel. Telephone: 97-248-294-136. Fax: 97-248-294-599. Email: noab@tx.technion.ac.il.

Received: March 3, 2012

DOI : $10.5430 / j b g c . v 2 n 1 p 15$
Accepted: March 29, $2012 \quad$ Published: June 1, 2012

URL: http://dx.doi.org/10.5430/jbgc.v2n1p15

\section{Abstract}

Background: Myocardial infarction (MI) is known to progress from the inner layers towards the epicardium. Since it is important to detect MI early, to prevent a negative remodeling process of the left ventricle (LV), the hypothesis of this study was that evaluation of layer-specific strains is feasible, and it will enable differentiation between subjects with large MI, small MI and normal LV.

Methods: In this study a commercial speckle tracking echocardiography (STE) program was modified to measure the strains at three myocardial layers instead of for the total-wall-thickness. After a validation process by using software implemented phantoms, the commercial and modified programs were applied to echocardiography of 54 subjects.

Results: The validation study results for 972 segments showed an agreement between the endocardial strains, manually measured by the commercial program, and automatically measured by the modified program. Finally, the algorithm was applied to scans of 15 normal subjects, 9 patients with small MI and to 6 patients with large MI. The results show that the strain elevated from the endocardium towards the epicardium for the normal and small MI groups, but not for the large MI group.

Conclusions: In conclusion, the layer-specific STE method allows accurate analysis of the transmural variations of the strains.

\section{Key words}

Speckle tracking echocardiography, Transmural variations, Strain imaging, Left ventricular function, Myocardial infarction

\section{I ntroduction}

Quantitative, accurate and reproducible analysis of the left ventricle (LV) mechanical function is of major importance in patients with coronary artery disease. Presently, the analysis is mainly based on tagged magnetic resonance imaging (MRI) and on echocardiography. Tagged-MRI is a non-invasive and efficient imaging modality that allows quantitative tissue motion estimation $^{[1,2]}$. Tagged images appear with a spatially encoded pattern that moves with the tissue and can therefore 
be analyzed so as to quantify the motion of the myocardium, in terms of measures of the local contractile performance, such as strain. The disadvantages of tagged-MRI are its relatively low temporal and spatial resolutions, the decay of the tagging along the cardiac cycle, and the very high costs of the equipment and its usage.

Until recently, the most common practice of assessing myocardial function by echocardiography has been limited to visual inspection of wall motion by experienced readers. This approach has several limitations, mainly low accuracy and low reproducibility, as evidenced for example, by the significant inter-observer variability ${ }^{[3]}$.

Several quantitative techniques for ultrasonic myocardial strain analysis have been developed in recent years. One of these is the Doppler-based tissue velocity imaging, which allows quantitative wall motion analyses, including calculation of strain and strain rate ${ }^{[4,5]}$. This technique, however, has several limitations that prevent its widespread clinical application. For one, the calculated velocities depend on the angle between the ultrasound beam and the actual myocardial velocity vector. True velocity often cannot be measured, and actually in some regions it cannot be measured at all. Additionally, a strain image of the entire LV requires a meticulous point by point calculation.

Perhaps the most promising methods of measuring myocardial displacements are based on speckle tracking echocardiography (STE) in 2D ultrasound images. STE do not suffer from the angle dependency associated with the Doppler-based tissue velocity imaging methods, thus they may be used in all myocardial regions and for a broader spectrum of patients. One of the most vastly used techniques is the 2D Strain (EchoPACTM, GE Healthcare Inc., Norway). This commercial tool employs tracking of speckled patterns within the gray scale demodulated images ${ }^{[6]}$. It allows automated analysis of the strain, throughout the entire LV cross-section images. The strain measurements, calculated by the $2 \mathrm{D}$ strain, were validated against different modalities ${ }^{[7-13]}$, and were found to be reliable and robust relatively to visual inspection of wall motion by experienced readers ${ }^{[14]}$. Similar to the visual examination of the heart, as performed by echocardiographers, the 2D-Strain method is based on an assumption of transmural uniformity of the longitudinal displacements across the myocardial wall, and thus the 2D Strain is incapable of distinguishing transmural variations. Following these transmural variations, however, is required when addressing important clinical issues like transmurality of MI or subendocardial ischemia.

This article presents an extension of the 2D-Strain, which will allow calculation of velocities and strains in three distinct layers across the myocardial wall. The extension, presented here, maintains some of the assumptions of the previous method, i.e. overall smoothness of the motion as well as smoothness of the shape of the region of interest (ROI), in both space and time. The main difference is however, the elimination of the assumption regarding the uniformity of the longitudinal motion (constant velocity and strain) across the LV wall. The myocardium is now modeled by three smooth chains of knots, for achieving strain measurements for 3 layers, instead of the single smooth chain used in the previous approach that provided results for the total wall thickness. This study is a validation analysis of the layer-specific algorithm, by using software implemented phantoms and by comparing the endocardial strain manually measured by the commercial program to that automatically measured by the algorithm proposed here. Finally, the layer-specific strains of normal LV and injured LV due to small and large MI are presented.

\section{Subjects and methods}

The algorithm consists of two main parts: (1) Definition of the ROI at each frame (The ROI separates the LV myocardial zone from the rest of the image); (2) Calculation of the displacements and strains at all points within the ROI in every frame. A correlation based tracking algorithm is employed in order to compute the frame to frame displacement of each myocardial point. This defines an initial "noisy" displacement field due to errors of the tracking. This field is then smoothed using two main physiology based assumptions: (A) the displacement field is a continuous smooth function of both space and time; (B) the myocardium is of a relatively smooth shape. The algorithm is described schematically in the block diagram in Figure 1. A detailed description of each of the steps of the algorithm is given below. 


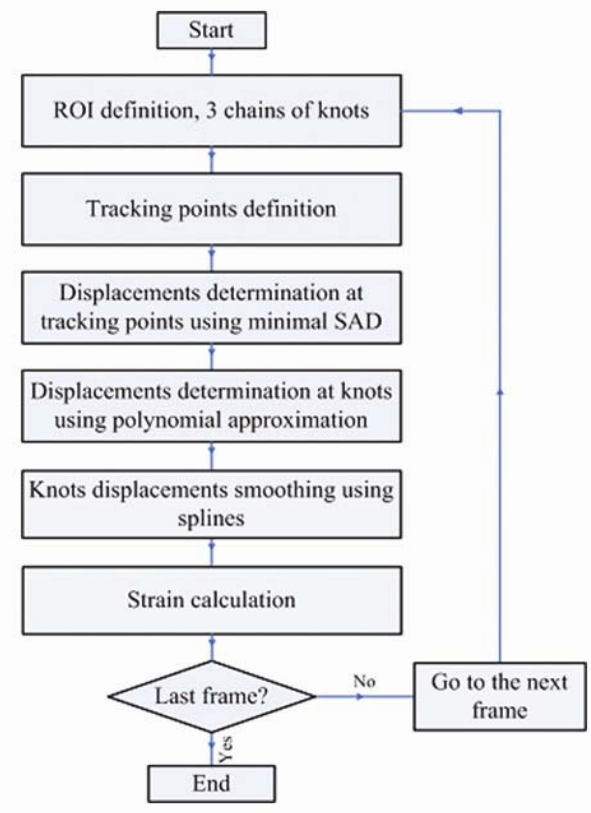

Figure 1. The block-scheme of the new layer-specific 2D-Strain algorithm. ROI=region of interest. SAD=sum of absolute difference.

A

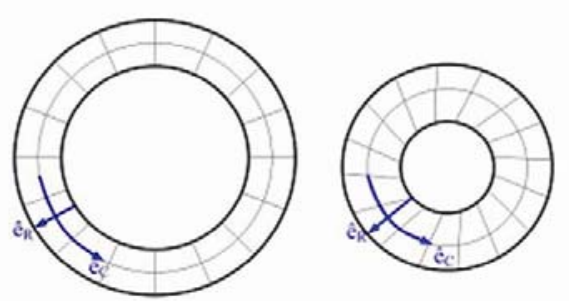

B

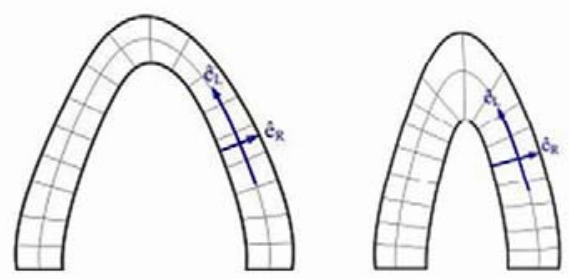

Figure 2. Short axis views, with the circumferential and radial directions marked. The typical non-uniform transmural contraction and rotation during the diastole (left) and systole (right) are shown (A). Long axis views, with the longitudinal and radial directions marked. The typical radial and longitudinal non-uniform contraction during the diastole (left) and systole (right) are shown (B).

\section{Definition of the ROI, degrees of freedom (3 chains of knots) and the deformation field:}

The ROI has two boundaries: an inner boundary, close to the endocardium, and an outer boundary, close to the epicardium. These boundaries are smooth curves defined by two chains of points (knots). These knots are further hypothesized to represent the same physical myocardial points at all frames. This results in a ROI that changes its shape from frame to 
frame. In order to enable the calculation of the radial myocardial deformations and strains, an additional chain of knots was placed midway between the two boundaries (see Figure 2 and 3). The three chains of knots describe the ROI global motion and deformation as a function of time. The coordinates of the knots as function of frame number $n_{\text {frame }}=1, \ldots, \mathrm{N}$, given by:

$$
X_{1}^{(n)}\left(n_{\text {frame }}\right), X_{2}^{(n)}\left(n_{\text {frame }}\right), X_{3}^{(n)}\left(n_{\text {frame }}\right), Y_{1}^{(n)}\left(n_{\text {frame }}\right), Y_{2}^{(n)}\left(n_{\text {frame }}\right), Y_{3}^{(n)}\left(n_{\text {frame }}\right) ; \quad n=1, \ldots, N
$$

constitute the $6 N$ degrees of freedom (independent functions of time) in terms of which the problem is completely defined.

The displacement field has been hypothesized to be spatially smooth. Therefore, the displacements $\Delta x, \Delta y_{\text {of an arbitrary }}$ point inside the ROI $(x, y)$ between every two frames can be expressed as a quadratic function of the coordinate differences between this point and the nearest knot $\left(x_{k n o t}, y_{k n o t}\right)$ :

$$
\begin{aligned}
& \Delta x=\Delta x_{\text {knot }}+A_{x}\left(x-x_{\text {knot }}\right)+B_{x}\left(y-y_{\text {knot }}\right)+C_{x}\left(x-x_{\text {knot }}\right)^{2}+D_{x}\left(y-y_{\text {knot }}\right)^{2}+E_{x}\left(x-x_{\text {knot }}\right)\left(y-y_{\text {knot }}\right) \\
& \Delta y=\Delta y_{\text {knot }}+A_{y}\left(x-x_{\text {knot }}\right)+B_{y}\left(y-y_{\text {knot }}\right)+C_{y}\left(x-x_{\text {knot }}\right)^{2}+D_{y}\left(y-y_{\text {knot }}\right)^{2}+E_{y}\left(x-x_{\text {knot }}\right)\left(y-y_{\text {knot }}\right)
\end{aligned}
$$

Here the displacements are defined as the coordinate differences in consecutive frames:

$$
\begin{array}{ll}
\Delta x=x\left(n_{\text {frame }}+1\right)-x\left(n_{\text {frame }}\right) & \Delta x_{\text {knot }}=x_{\text {knot }}\left(n_{\text {frame }}+1\right)-x_{\text {knot }}\left(n_{\text {frame }}\right) \\
\Delta y=y\left(n_{\text {frame }}+1\right)-y\left(n_{\text {frame }}\right) & \Delta y_{\text {knot }}=y_{\text {knot }}\left(n_{\text {frame }}+1\right)-y_{\text {knot }}\left(n_{\text {frame }}\right)
\end{array}
$$

The coefficients $A_{x}, B_{x}, C_{x}, D_{x}, E_{x}, A_{y}, B_{y}, C_{y}, D_{y}, E_{y}$ are in units of strain rates and spatial derivatives of the strain rates.
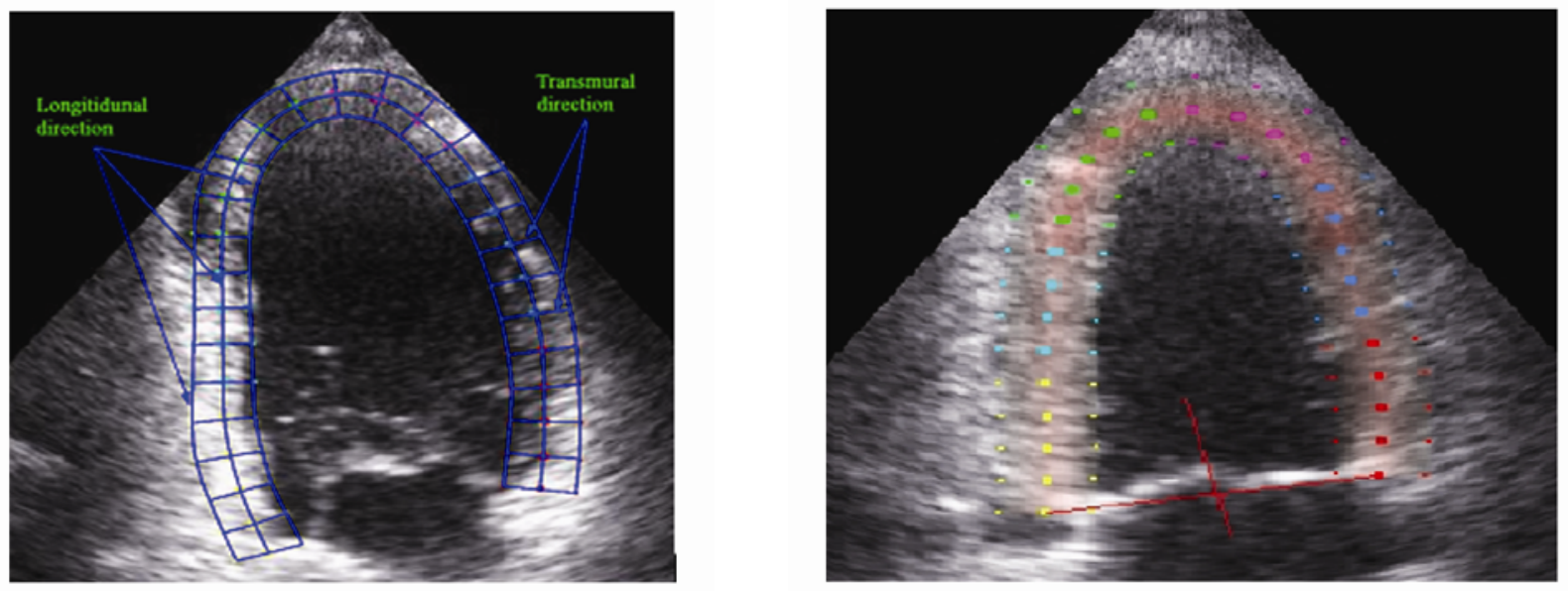

Figure 3. The definition of the degrees of freedom assumed by the new layer-specific 2D-Strain algorithm, depicted for end-diastole. There are 3 lines of knots that define the ROI.

In the first frame, the ROI internal boundary and the ROI width are defined automatically by the program such that it includes the main portion of the myocardial wall. In the consecutive frames the ROI boundaries and the mid-ROI chain of 
knots are modified automatically using the frame to frame tracking results and an appropriate, mainly spatial filtering scheme.

\subsection{Tracking and smoothing the displacements of the knots}

The displacement of an arbitrary point between two subsequent frames is assessed using a criterion of minimal sum of absolute differences (SAD), as described by Rappaport et al. ${ }^{[6]}$. Due to measurement noise, speckle noise, out-of-plane probe motion as well as other reasons ${ }^{[6]}$, the resulting displacement field is noisy and irregular. An example of an unsmoothed displacement field is shown in Figure 4A.

A

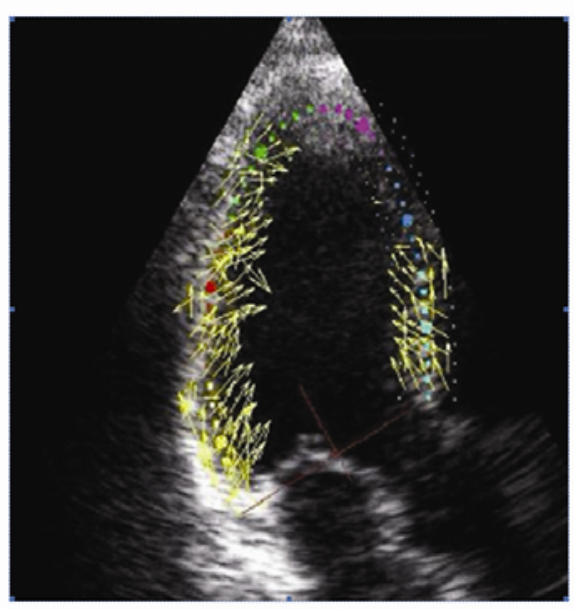

B

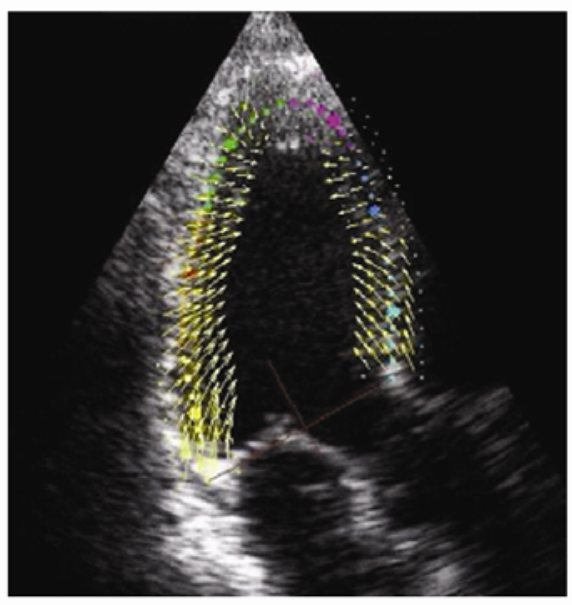

Figure 4. Non-smoothed (A) and smoothed (B) displacements fields

In order to further minimize the effects of the various noise factors, a multitude of points around each knot ("tracking points") is selected. Each tracking point represents a tracking kernel, which is a square segment of the image. The kernel has to be significantly larger in size than a typical speckle in order to ensure effective correlation tracking. At the same time, the kernel should be small enough so that all its points have nearly the same displacement. A reasonable typical size is $\sim 20 * 20$ pixels (a few millimeters in a regular echo image). The kernel size was optimized experimentally, and it was found that small variations do not affect the tracking results. There is also a $50 \%$ overlap between adjacent tracking kernels. The overall number of tracking points is proportional to the length and width of the ROI. A typical number of tracking points is $\sim 100-200$ along the ROI and 4 across. As mentioned above, the displacement of each tracking point is computed using a minimum SAD algorithm. As a rule, the search region must be larger than the maximal possible tissue displacement between two consecutive frames. The size of the search area is roughly inversely proportional to the frame rate. For a typical human echocardiogram at a moderate frame rate $(\sim 50 \mathrm{fps})$, it is around $5 \mathrm{~mm}$. Assuming spatial smoothness of the displacement field, the displacements of the tracking points can be determined in terms of a small number of parameters. The resultant over-determined system of equations is solved using a least square method. Details of the calculations are given in Appendix 1. The resultant smoothed velocity field is now used for the calculations of the displacements and new positions of the knots.

In order to further reduce the effect of noise on the calculation of the displacements, it is assumed that the variations in the longitudinal and circumferential directions are smooth enough so that the characteristic spatial variation size is about a few millimeters. This assumption is supported by previous clinical results ${ }^{[15]}$. According to this assumption, the displacements in all 3 chains may be smoothed, using a smoothing spline. The results of such a two-stage smoothing procedure create a local smoothing of the velocity around each knot and a global smoothing of the velocity of the entire chain of knots (see Figure 4B). 


\subsection{Strain calculation}

When the displacement field is known, the strain tensor components can be calculated by:

$$
\begin{aligned}
& \varepsilon_{X X}=\frac{\partial \Delta X\left(n_{\text {frame }}\right)}{\partial X(1)} ; \quad \varepsilon_{Y Y}=\frac{\partial \Delta Y\left(n_{\text {frame }}\right)}{\partial Y(1)} \quad \varepsilon_{X Y}=\varepsilon_{Y X}=\frac{1}{2}\left(\frac{\partial \Delta X\left(n_{\text {frame }}\right)}{\partial Y(1)}+\frac{\partial \Delta Y\left(n_{\text {frame }}\right)}{\partial X(1)}\right) \\
& \Delta X\left(n_{\text {frame }}\right)=X\left(n_{\text {frame }}\right)-X(1) ; \Delta Y\left(n_{\text {frame }}\right)=Y\left(n_{\text {frame }}\right)-Y(1)
\end{aligned}
$$

Here the strain is calculated at the point $X(1), Y(1)$ in frame number 1 , and consequently at the point $X\left(n_{\text {frame }}\right), Y\left(n_{\text {frame }}\right)$ in frame $n_{\text {frame }}$. The displacement of the knots between the initial frame and frame $n_{\text {frame }}$ can be large, thus before the numeric calculation of derivatives of equation (3) is performed, the displacement field needs to be spatially interpolated. A cubic spline interpolation is used in the longitudinal and circumferential directions. The displacement field is assumed to be almost a linear function of the radial coordinate, due to the relatively small width of the heart wall. Therefore the parabolic interpolation was used along the radial direction.

After calculation of the displacement of the knots, new knot coordinates are defined for the new frame, and the algorithm returns to the first stage. When the last frame is reached, the algorithm stops.

\subsection{Rotation calculation}

The knot's angular shift between subsequent frames is calculated as the amount of linear displacement in the direction orthogonal to the radius of the knot, divided by the size of this radius as described by Notomi et al. ${ }^{[1]}$. The radius is the distance of the knot from the immediate geometrical center of entire chain of knots.

\subsection{Evaluation of the algorithm}

\subsubsection{Software implemented phantoms}

A set of software implemented phantoms was designed and employed for the validation of the algorithm. The first phantom was produced using the Field II program ${ }^{[16-18]}$. The program generates a simulated B-scan image for a given spatial distribution and a predetermined set of reflection coefficients of scatters. The ultrasonic parameters were selected to be similar to the usual setting selected in an echocardiography study, and they are listed in the Table 1.
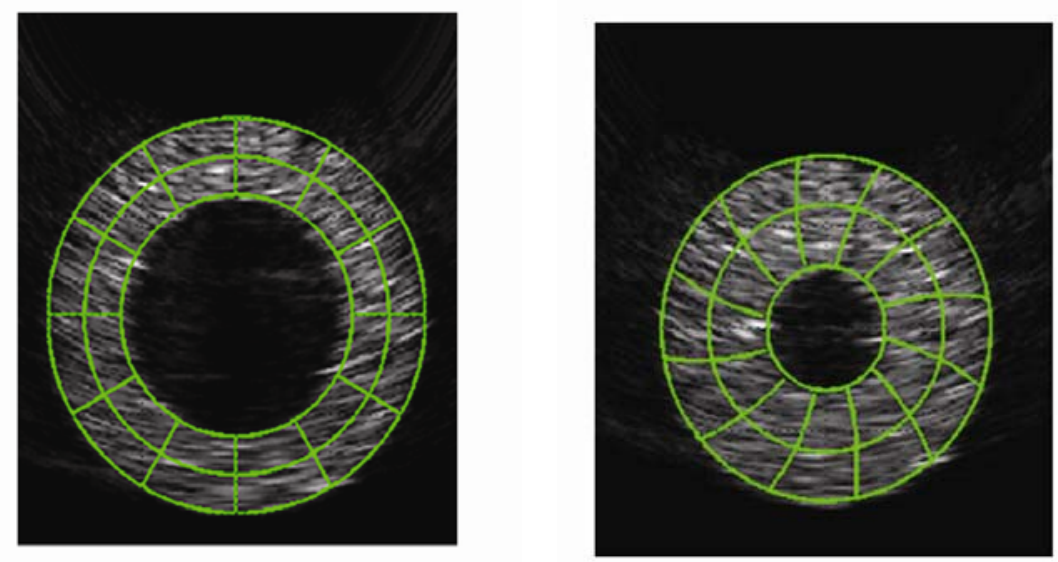

Figure 5. Images generated by Field II at diastole (left) and at systole (right), with schematic presentation of the displacement field (green lines). 
Table 1. Set-up for Field II phantom

\begin{tabular}{ll}
\hline Transducer parameters & \\
Width of element & $0.375 \mathrm{~mm}$ \\
Height of element & $7 \mathrm{~mm}$ \\
Distance between elements & $0.025 \mathrm{~mm}$ \\
Number of physical elements & 64 \\
Focus (fixed focal point) & $70 \mathrm{~mm}$ \\
Scan setting & \\
Center frequency & $2 \mathrm{MHz}$ \\
Speed of sound & $1540 \mathrm{~m} / \mathrm{s}$ \\
Wavelength & $0.77 \mathrm{~mm}$ \\
Sampling rate & $6 \mathrm{MHz}$ \\
Scan sector & $\mathrm{pi} / 2$ \\
Number of scan lines & 64 \\
\hline
\end{tabular}

The scatters in each frame are located within a cylindrical volume. There are about $10^{5}$ small scatters and about $10^{3}$ strong scatters in the region with $85 \mathrm{~mm}$ width, $85 \mathrm{~mm}$ depth and $10 \mathrm{~mm}$ height. The reflectivity of the strong scatters is 100 times larger than the reflectivity of the weak Raleigh scatters. Two examples of frames from a clip are presented in the Figure 5.

The deformations of the cylindrical-shaped phantom generated by the Field II program (see Table 2) consist of a combination of non-uniform contractions and rotations in the radial direction, mimicking similar deformations of the apical short axis of the heart (see Figure 2A). The deformations are defined in such a way that the cross-sectional area of the 'myocardium' is always preserved, simulating conservation of mass. The rotation linearly increases with the radius. Concurrent with the time-dependent deformations, the positions of the scatterers are changing, thus a different spatial distribution of scatterers is generated for each new B-scan image. The numerical values of the contraction and rotation were used to generate the typical values of radial strain, circumferential strain and shear of healthy persons ${ }^{[15]}$. There was no out-of-plane motion. These simulations allowed studying the accuracy of the algorithm in the presence of speckle noise.

Table 2. Results of tracking for Field II phantom

\begin{tabular}{lll}
\hline & Imposed value & Calculated value \\
\hline$R_{\text {in }}$, systole, mm & 12 & $14 \pm 2$ \\
$R_{\text {mid }}$, systole, mm & 23 & $24 \pm 2$ \\
$R_{\text {ext }}$, systole, mm & 34 & $34 \pm 2$ \\
$\varphi_{\text {in }}$, systole, deg & 20 & $20 \pm 2$ \\
$\varphi_{\text {mid }}$, systole, deg & 15 & $14 \pm 1$ \\
$\varphi_{\text {ext }}$, systole, deg & 10 & $11 \pm 1$ \\
Peak inner circumferential strain & $-50 \%$ & $-49 \pm 3 \%$ \\
Peak mid circumferential strain & $-25 \%$ & $-26 \pm 2 \%$ \\
Peak outer circumferential strain & $-14 \%$ & $-13 \pm 2 \%$ \\
Peak mean radial strain & $27 \%$ & $35 \pm 2$ \\
\hline
\end{tabular}

Note. $R$ is the radius and $\varphi$ is the maximal rotation angle. 


\subsubsection{Validation of the endocardial strain measurement}

The second stage of validation was a comparison between the endocardial longitudinal strain that was measured by the layer-specific 2D-strain program and the endocardial longitudinal strain that was measured by applying a manual narrow ROI at the inner layers of the myocardium by using the 2D-strain. For that purpose we used long-axis views ( 2 chambers, 4 chambers and 5 chambers) from 54 human subjects. Echocardiography study was performed by using Vivid 7 system (GE Healthcare, Inc., Horten, Norway) with the standard 3S probe and with frequency of $3 \mathrm{MHz}$ and frame rates in excess of 40 frames per second (in order to ensure tracking). For the validation study, all patients gave their informed consent for the anonymous use of the results of their echocardiographic examinations. The study was performed according to the regulations of the ethics committee of Kaplan medical center, Rehovot, Israel.

\subsection{Clinical examples}

The method presented here allows evaluation of typical parameters of cardiac muscle deformation such as circumferential strain in the endocardial and epicardial regions. These parameters can have a potential diagnostic value, e.g. in the diagnosis of non-transmural infarctions as well as of other non-transmural cardiac pathologies. To illustrate the potential diagnostic value of the layer-specific parameters, the segmental LV rotation and peak systolic strain (PSS) were calculated. The PSS is a widely accepted parameter in the analysis of segmental deformations in acute myocardial infarctions (AMI), which describes maximum contraction during systole ${ }^{[19-21]}$. Two groups were examined: healthy subjects $(\mathrm{n}=15)$ and patients $(\mathrm{n}=15)$ with infarctions involving the apical zones (LAD). For each group, values of these parameters were computed in the apical short axis view. Measurements were performed on Vivid 7 system (GE Healthcare, Inc., Horten, Norway) with the standard $3 \mathrm{MHz}$ probe and with frame rates in excess of 40 frames per second (in order to ensure tracking).

The definition of an AMI followed the Guidelines of the European Society of Cardiology, including patient history, the 12 lead ECG reading, Creatine Phosphokinase (CPK) level and coronary angiography.

Being a retrospective study in nature, its results did not affect medical management of the patients in any way. All patients and individuals of the control group gave their informed consent for the anonymous use of the results of their echocardiographic and heart catheterization examinations for this study. The study was performed according to the regulations of the ethics committee of the St. Vinzenz-Hospital, Köln.

\subsection{Statistical analysis}

All values are presented as mean \pm standard deviation. For the comparison between the endocardial strain, calculated automatically by the layer-specific $2 \mathrm{D}$ strain, and the endocardial strain, calculated by a manual narrow ROI, the Bland-Altman statistical analysis was used ${ }^{[22]}$. For the clinical examples, the comparison between the endocardial strain, mid-wall strain and epicardial strain was performed by students' $t$-test.

\section{Results}

\subsection{Simulations by software implemented phantoms}

The values of the deformation parameters calculated by the method described above were compared to those used for the definition of the Field II software implemented phantom and the results are given in Table 2. The strain tensor components were calculated in polar coordinates. Explanations for the calculations are presented in Appendix 2. The results show that the layer-specific 2D-strain program estimates the rotation and circumferential strain with rather small errors (1deg of rotation and $1 \%$ of strain) between the measured values and the imposed values. However, the radial strain is measured with larger errors of $7 \%$ strain. 


\subsection{Validation of the endocardial longitudinal strain}

The endocardial longitudinal strain was automatically calculated by the layer-specific 2D-strain, and by a manual ROI that was applied to the endocardial border of the LV by using the commercial 2D-strain. The Bland-Altman results show a good agreement between the two methods (see Figure 6). When considering the apical, mid-ventricular and basal segments, the bias was $2.3 \%$, the upper limit of agreement was $8.9 \%$ and the lower limit of agreement was $-4.3 \%$ (see Figure $6 \mathrm{~A}$ ). When not taking into consideration the apical segments, the bias was $1.1 \%$, the upper limit of agreement was $5.5 \%$ and the lower limit of agreement was $-3.3 \%$ (see Figure $6 \mathrm{~B}$ ).

A

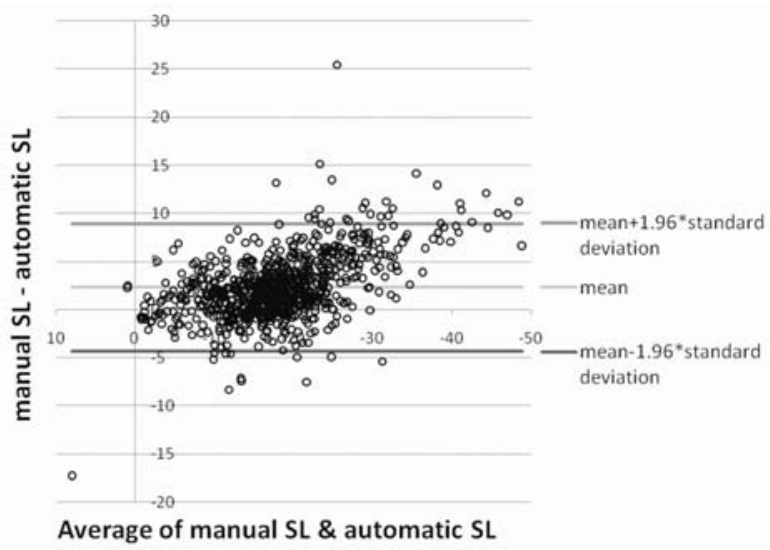

$\mathrm{B}$

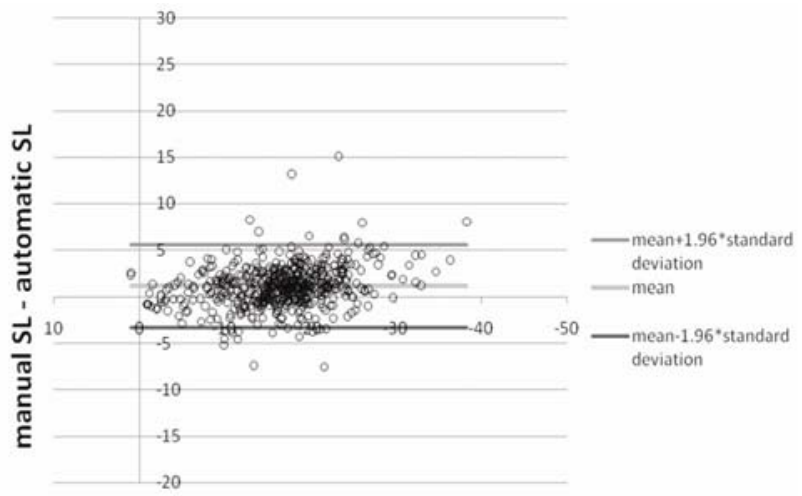

Average of manual SL \& automatic SL

Figure 6. Bland-Altman statistical analysis for assessing the difference between the segmental endocardial longitudinal strains, calculated automatically by the layer-specific 2D-strain program, and the endocardial longitudinal strains, calculated after a manual definition of a narrow ROI by using the commercial 2D-strain. The analysis was performed once for the results for the apical, mid ventricular and basal segments (A) and once without considering the apical segments (B)

\subsection{Clinical examples}

Table 3. Experimental results of clinical study. A control group $(n=15)$ compared with a group of patients $(n=15)$, suffering from small $(\mathrm{n}=9$ and large $(\mathrm{n}=6)$ acute LAD infarctions. Values are presented as average standard deviation.

\begin{tabular}{llll}
\hline Group & Healthy & Small LAD & Large LAD \\
\hline Endocardial peak circumferential strain, \% & $-41 \pm 8$ & $-29 \pm 7 \#$ & $-11 \pm 6 \dagger$ \\
Mid-wall peak circumferential strain, \% & $-31 \pm 6$ & $-22 \pm 5^{*}$ & $-8 \pm 4 \ddagger$ \\
Epicardial peak circumferential strain, \% & $-24 \pm 5$ & $-17 \pm 5^{* *}$ & $-6 \pm 3 \ddagger$ \\
Endocardial peak longitudinal strain, \% & $-34 \pm 4$ & $-18 \pm 6^{* * *}$ & $-8 \pm 9 \dagger$ \\
Midwall peak longitudinal strain, \% & $-25 \pm 4$ & $-11 \pm 4^{* * *}$ & $-4 \pm 6 \dagger$ \\
Epicardial peak longitudinal strain, \% & $-18 \pm 3$ & $-6 \pm 2 * * *$ & $-2 \pm 5 \dagger$ \\
Peak rotation, deg & $8 \pm 5$ & $10 \pm 7$ & $4 \pm 9$ \\
\hline
\end{tabular}

$\# P<0.05$ vs. Healthy, ${ }^{*} P<0.01$ vs. Healthy, ${ }^{* *} P<0.001$ vs. Healthy, ${ }^{* * *} P<0.0001$ vs. Healthy. ${ }^{\dagger} P<0.05$ vs. small LAD, $¥ P<0.001$ vs. small LAD.

In order to demonstrate the potential clinical relevance of the proposed method, we applied it to data acquired from 15 normal subjects and 15 patients with infractions involving the apex (LAD). Some of the infarctions $(n=6)$ were most likely transmural, based on the clinical assessment and blood biomarkers and the others $(n=9)$ were most likely non-transmural. Typical images and traces for each of the cases are shown in Figures 7-9. Each of the Figure shows the circumferential strain results for 3 myocardial layers and 6 segments (A) for a short axis view (apical level), and the layer-specific longitudinal strain for 3 layers and 6 segments of one of the apical long axis views (B). Rotation (C) for each of these 6 segments are also demonstrated in Figures 7-9. The results detailing the peak circumferential strain, peak longitudinal strain and rotation, are summarized in Table 3. As seen in this table, the values of the strains are different for healthy 
people and patients ( $P$ values are stated at the table), while the values of rotation are the same. Furthermore, peak systolic circumferential and longitudinal strains are significantly different between the layers for the normal group (circumferential $P<0.01$, longitudinal $P<0.0001)$ and small LAD group $(P<0.05)$, but not for the large LAD group.

A

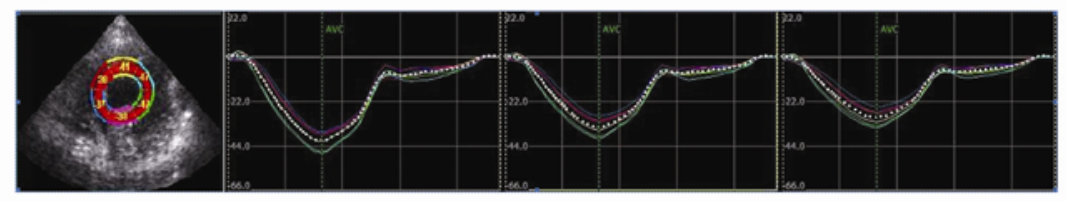

$\mathrm{B}$

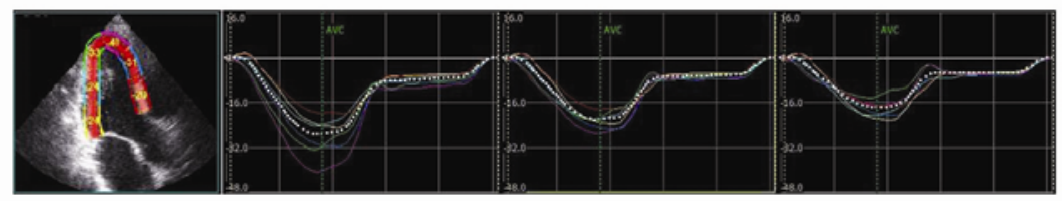

C
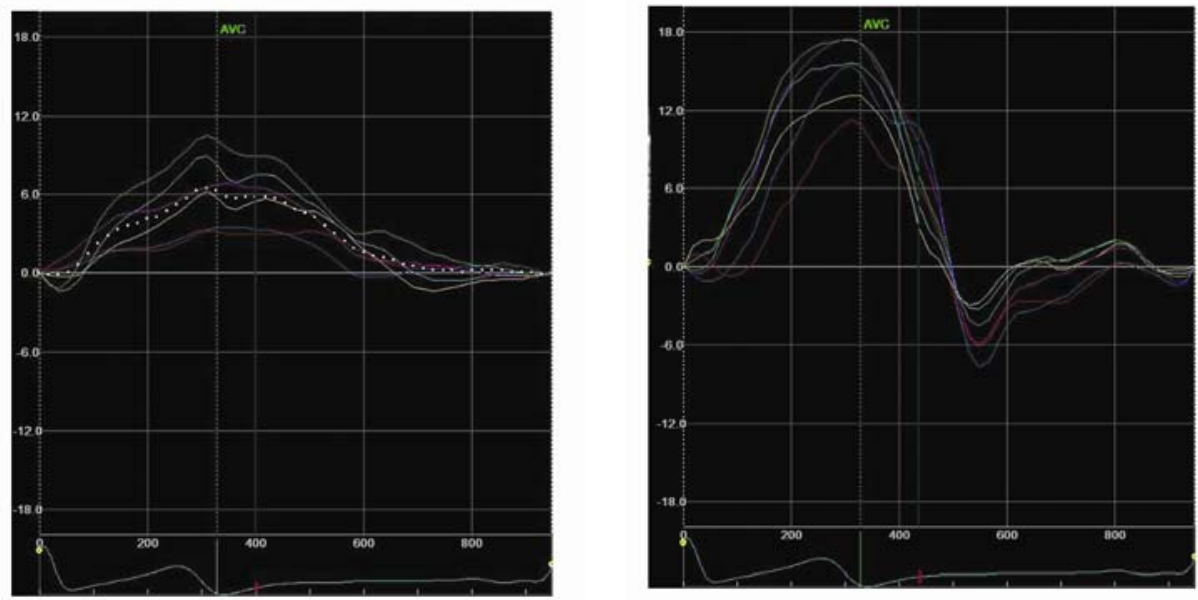

Figure 7. Normal subject. Segmental Circumferential (A) and Longitudinal (B) Strain traces in the subendocardial, mid-wall and subepicardial layers and segmental rotation traces $(\mathrm{C})$

\section{Discussion}

The algorithm described above is an extension of the commercial 2D-Strain speckle tracking program, described by Rappaport et al. ${ }^{[6]}$. 2D-Strain has a number of advantages in the routine clinical practice and is also important in clinical and physiological research ${ }^{[12,14]}$. The layer-specific 2D-Strain algorithm, presented here, substantially extends these capabilities. The results of this study show that: 1) It is possible to reliably assess layer-specific strains in 3 myocardial layers; 2) The layer-specific 2D strain enables the differentiation between normal LV, LV with small MI and LV with large MI.

One of the limitations of the commercial 2D-Strain algorithm is that strain variations across the myocardial wall are averaged out. Thus, important physiological and clinical quantities, like layer-specific strains, cannot be evaluated. Indeed, there are cases in which only the epicardial layer of the myocardium is functioning properly or even compensating for the impaired inner layers ${ }^{[23]}$. Such compensation might result in almost normal average strain values. On the other hand, other transmural strain attributes, such as layer-specific strain, might be emphasized. Consequently, an important promising aspect of the layer-specific 2D-Strain is the potential feasibility of detecting subendocardial ischemia. 
Examples of clinical studies, in which the layer-specific 2D-strain algorithm was already used, are: 1) the study of Leitman et al. that described the layer specific strains in LV that suffer from wall motion abnormalities ${ }^{[24]}$; 2 ) the study of Becker et al. that described the layer specific strains in chronic patients that suffer from MI ${ }^{[25]}$; 3 ) the study of Leitman et al. that described the layer-specific strains in patients that suffer from myocarditis ${ }^{[26]}$.

A

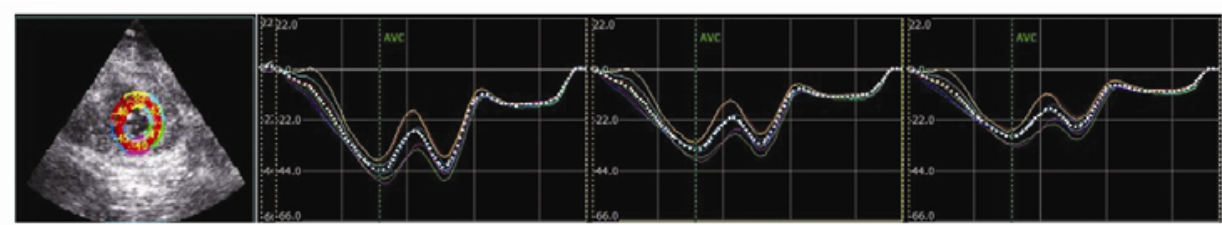

B

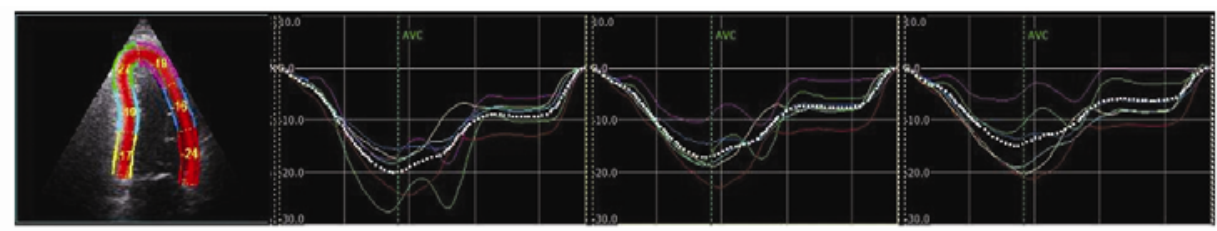

$\mathrm{C}$
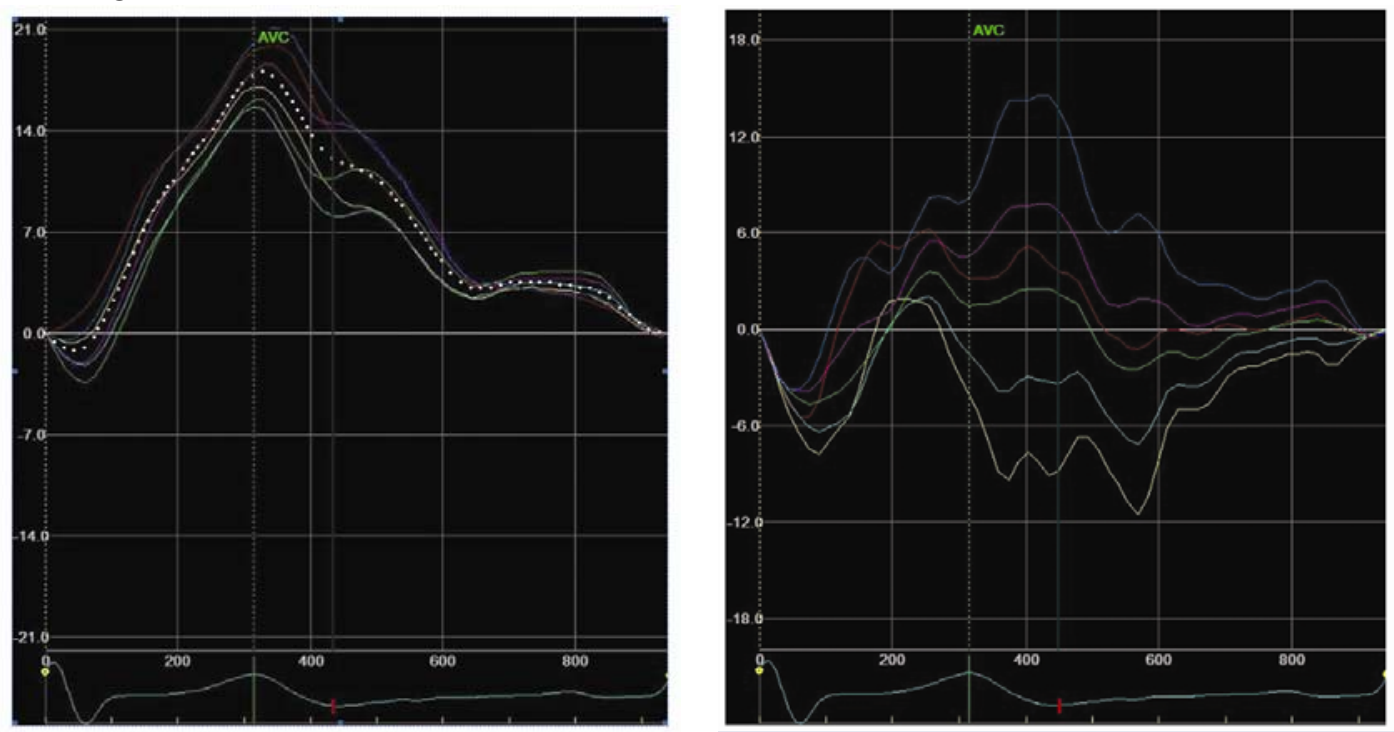

Figure 8. A patient with a small LAD infarction: Segmental Circumferential (A) and Longitudinal (B) Strain traces in the subendocardial, mid-wall and subepicardial layers and segmental rotation traces (C).

The evaluation of the performance of the layer-specific 2D-Strain algorithm on data produced by the software implemented phantoms allows prediction of the ranges of parameters in which the algorithm is applicable. Another advantage of these phantoms is the precise knowledge of the deformation parameters. Such information is generally not available in most in-vivo or in-vitro experiments, where the deformation field can only be roughly estimated ${ }^{[2]}$. In addition, the validation on the data produced by the software implemented phantoms also allows estimating the robustness of the method with respect to speckle noise. Speckle noise is one of the largest sources of errors in the tracking ${ }^{[27]}$. This might explain the small differences between the imposed deformation parameters and the tracking results. Analyses of the tracking results indicate that the errors in the circumferential strain and the errors in the mean radial strain are much smaller than their typical values in the normal human heart ${ }^{[2]}$. Consequently, the layer-specific 2D-Strain potentially can be used for measuring these quantities. 
A

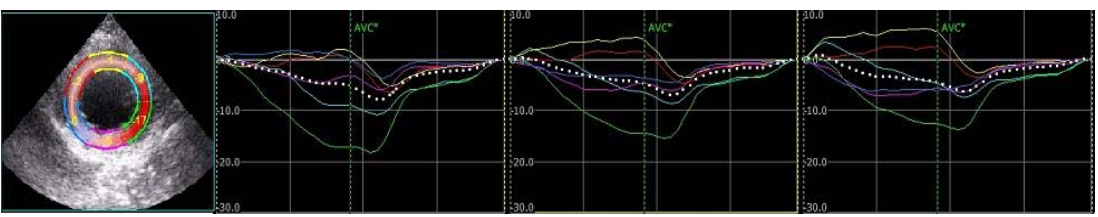

B

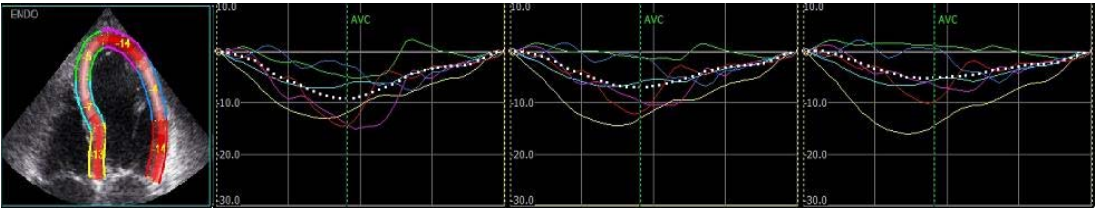

$\mathrm{C}$
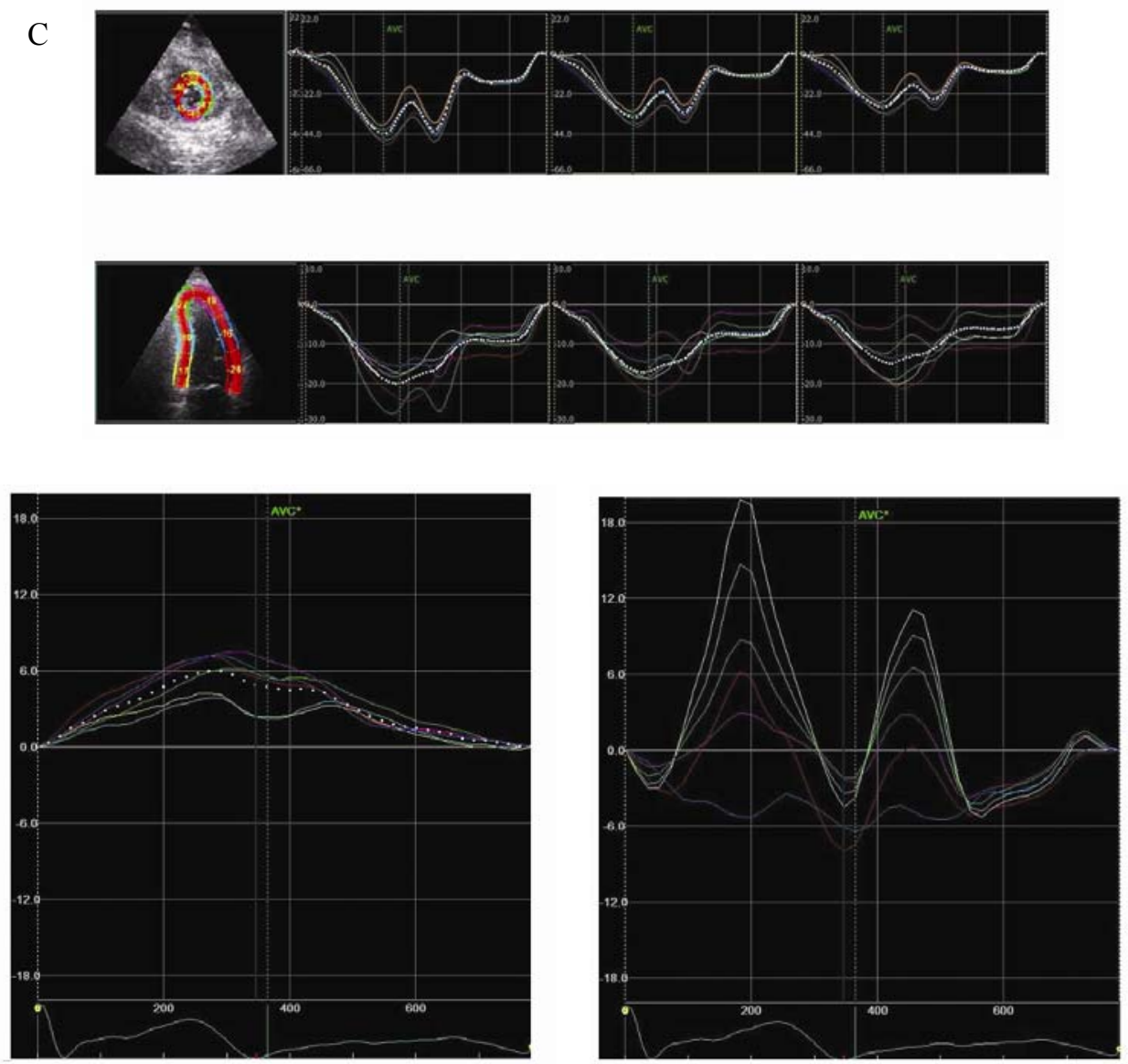

Figure 9. A patient with a large LAD infarction: Segmental Circumferential (A) and Longitudinal (B) Strain traces in the subendocardial, mid-wall and subepicardial layers and segmental rotation traces $(\mathrm{C})$.

Another possible source of error in the estimation of transmural deformation parameters could be the smoothing procedure being used. The smoothing is essentially one-dimensional, i.e. all the quantities are smoothed in circumferential or longitudinal directions, thus underestimation of the strain parameters occurs (see Table 2). In addition, the errors in evaluating the strain increase when the strain increases ${ }^{[27]}$. As shown in the Appendix 3, the deformations increase as the radius decreases, thus the values of strains in the inner layer are expected to be larger and less accurate. This suggests that the smoothing procedure can be improved by using smoothing parameters that are dependent on the transmural direction. However, in this study we showed that the endocardial longitudinal strain is a reliable parameter. This validation was performed by comparing the commercial 2D-strain results, when applying a manual narrow ROI to the endocardium, to the results of the layer-specific 2D-strain, when applying an automatic wide ROI to the whole myocardium. The results 
show a good agreement between the two methods (see Figure 6A), though a better agreement is shown when disregarding the apical results (see Figure 6B). The reason is that the error of the layer-specific strain measurements increases as the radius of curvature increases ${ }^{[28]}$. Moreover, the tracking quality is smaller in the apical location.

After the validation study, the new algorithm was applied to apical short axis scans of 15 normal subjects, of 9 patients with minor LAD acute infarctions and of 6 patients with large LAD infarction (see Figures 7-9, Table 3). It was shown that in these patients the peak systolic strain is markedly reduced in the ischemic segments compared with the non-ischemic remote regions ${ }^{[29]}$. Furthermore, it was observed that any size of an LAD infarction, even a small one, is inherently manifested as a decreased systolic contraction (negative circumferential strain) in all 3 myocardial layers. Thus, measurement sensitive to the differences among the layers across the myocardial wall may help in the diagnosis of MI. Non-transmurality is indicated by the persistence of systolic contraction in all 3 layers, as well as persistence of the circumferential (or longitudinal) strain gradient from the sub-epicardium to the sub-endocardium. In the transmural example depicted in Figure 9 the systolic contraction, contraction gradient and rotation are totally lost in some of the segments. Another interesting observation is the poly-phasic nature of the strain curves in the "likely non-transmural" LAD, which according to ex-vivo experiments could be an indication of non-transmural ischemia ${ }^{[30]}$. The well known layered structure of the cardiac walls is reflected in the shear parameter, whose behavior is not clear ${ }^{[31]}$.

\section{Limitation}

We have validated the method using Field-II simulations. Although the Field-II does simulate some of attributes affecting frame-to-frame tracking, such as motion-induced variations in speckle patterns, it does not simulate other sources of errors, e.g. out-of-plane motion, clutter noise and random noise. A second limitation is related to any two-dimensional speckle tracking of motion. The tracking strongly depends on the existence of "non-Rayleigh" reflectors and their persistence for more than two consecutive frames. Out of plane motion reduces the persistence of these reflectors. Another limitation exists in the short axis views, in which the deformation parameters are sensitive to the angle of the scanning plane. This is because tilting the plane of acquisition strongly affects the measured strain distribution around the short axis, due to the significant base-to-apex strain variations. The effect is of course very different from the well-known angle dependency of Doppler-based tissue velocity imaging, where the measured velocities depend on the angle between the ultrasonic beam and the direction of the velocity vector.

In conclusion, layer-specific, multilayer strain analysis is feasible, and may provide a better insight of myocardial pathologies as MI.

\section{Acknowledgement}

This study was supported by the "Magneton" project, Chief Scientist, Israel Ministry of Industry Trade and Labor, and by the Technion VPR Fund for the promotion of research.

\section{Source(s) of support}

This study was supported by the "Magneton" project, Chief Scientist, Israel Ministry of Industry Trade and Labor, and by the Technion VPR Fund for the promotion of research.

\section{Appendix 1. Least square polynomial approximation for knots displacementsEquation Section (Next)}

In the vicinity of every knot we choose $N_{\text {tracking }}$ tracking points with relative coordinates $\left(\Delta x_{n_{\text {maxding }}}, \Delta y_{n_{\text {nadading }}}\right)$. The displacements

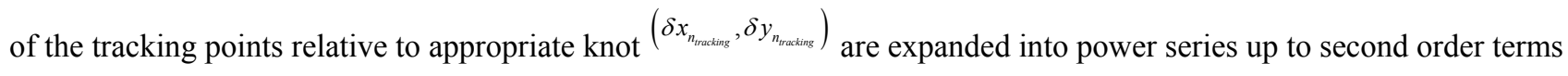


(A1). Due to presence of noise in order to find knots displacements as a function of tracking point's displacements, it is convenient to use the least square method. The cost function is:

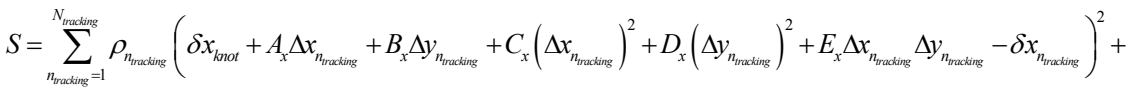

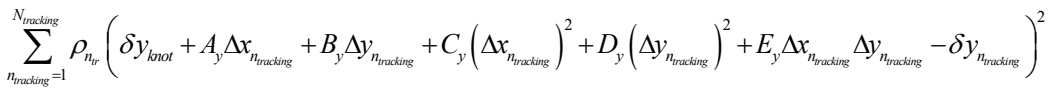

$$
\begin{aligned}
& \rho_{n_{t r}}, \sum_{n_{t r}} \rho_{n_{t r}}=1
\end{aligned}
$$

In the equation (A1) the numbers $\rho_{n_{t r}}, \sum_{n_{t r}} \rho_{n_{t r}}=1$ represent weights, associated with tracking quality of a given tracking point. Differentiating of the cost function $S$ with respect to parameters $\delta x_{k n o t}, \delta y_{k n o t}, A_{x, y}, B_{x, y}, C_{x, y}, D_{x, y}, E_{x, y}$ leads to the system of linear equations on these parameters.

\section{Appendix 2. Strain tensor in polar coordinates}

The length element in the polar coordinates $(r, \varphi)$ is:

$$
d l^{2}=d r^{2}+r^{2} d \varphi^{2}
$$

As a result of deformation point with coordinates $(r, \varphi)$ at frame number 1 transforms into the point $(\tilde{r}, \tilde{\varphi})$ at frame number $n_{\text {frame }}$ :

$$
\begin{aligned}
& \tilde{r}=r+\Delta r(r, \varphi) \\
& \tilde{\varphi}=\varphi+\Delta \varphi(r, \varphi)
\end{aligned}
$$

The strain tensor components in the polar coordinates $(r, \varphi)$ are defined in terms of new length element:

$$
\begin{aligned}
& d \tilde{l}^{2}=d l^{2}+2\left(s_{r r} \cdot d r^{2}+s_{r \varphi} \cdot r \cdot d r \cdot d \varphi+s_{\varphi \varphi} \cdot r^{2} \cdot d \varphi^{2}\right) \\
& s_{r r}=\frac{\partial \Delta r}{\partial r} ; \quad s_{r \varphi}=\frac{1}{2}\left(r \frac{\partial \Delta \varphi}{\partial r}+\frac{1}{r} \frac{\partial \Delta r}{\partial \varphi}\right) ; \quad s_{\varphi \varphi}=\frac{\partial \Delta \varphi}{\partial \varphi}+\frac{1}{r} \Delta r
\end{aligned}
$$

The component $S_{r r}$ represents radial strain; the component $S_{r \varphi}$ represents shear and the component $S_{\varphi \varphi}$ represents circumferential strain. In order to evaluate radial strain and shear and to reduce the effect of noise, the displacements $\Delta r(r, \varphi), \Delta \varphi(r, \varphi)$ are approximated by a linear function of the radial coordinate $r$. Under this assumption the radial strain does not depend on radial coordinate. Therefore the assumption is equivalent to averaging the radial strain over all 3 chains of knots. The displacements dependence on circumferential coordinate $\varphi$ is assumed to be a quadratic function of the radial coordinate.

\section{Appendix 3. Circumferential strain variations in cylindrical approximation}

Below we analyze the circumferential strain variations in the case of radially contracting hollow cylinder with an incompressible wall. Let the radii of the internal ("endocardial") and external ("epicardial") wall borders be $R_{\text {end }}(t)$ and $R_{e p i}(t)$, respectively. The circumferential Lagrangian strain Sc at each border depends on the temporal variation of the border length $\mathrm{L}$ (or its radius $R$ ):

$$
S_{C}(t)=\frac{L(t)-L(0)}{L(0)}
$$

The circumferential strains in both wall borders are thus: 


$$
S_{\text {Cend }}(t)=\frac{R_{e n d}(t)-R_{e n d}(0)}{R_{e n d}(0)} \quad \text { and } \quad S_{\text {Cepi }}(t)=\frac{R_{e p i}(t)-R_{e p i}(0)}{R_{e p i}(0)}
$$

The mass conservation of the wall transforms into constant wall area, which in symmetric case reads:

$$
R_{e p i}^{2}(t)-R_{e n d}^{2}(t)=\text { const }=R_{e p i}^{2}(0)-R_{e n d}^{2}(0)
$$

After a simple combination of these equations we get the relation between the endocardial and epicardial circumferential strain:

$$
S_{\text {Cend }}(t) \cdot\left(S_{\text {Cend }}(t)+2\right)=\left(\frac{R_{\text {epi }}(0)}{R_{\text {end }}(0)}\right)^{2} \cdot S_{C e p i}(t) \cdot\left(S_{C e p i}(t)+2\right)
$$

The solution of this quadratic equation is:

$$
S_{\text {Cend }}(t)=-1+\sqrt{1+\left(\frac{R_{e p i}(0)}{R_{\text {end }}(0)}\right)^{2} \cdot S_{\text {Cepi }}(t) \cdot\left(S_{\text {Cepi }}(t)+2\right)}
$$

In this context, one can define the endocardial strain gain as the ratio between endocardial and epicardial strains.

\section{References}

[1] Moore CC, Lugo-Olivieri CH, McVeigh ER, Zerhouni EA. Three-dimensional systolic strain patterns in the normal human left ventricle: characterization with tagged MR imaging, Radiology. 2000; 214: 453-466. PMid:10671594

[2] Zerhouni EA, Parish DM, Rogers WJ, Yangand A, Shapiro EP. Human heart: tagging with MR imaging - a method for non-invasive assessment of myocardial motion, Radiology. 1988; 169: 59-63. PMid:3420283

[3] Bolger A. Do you see what I see? Agreeing to disagree. J Am Coll Cardiol. 2006; 47: 129-30. PMid:16386675 http://dx.doi.org/10.1016/j.jacc.2005.10.013

[4] Stoylen A, Slordahl S, Skjelvan GK, Heimdal A, Skjaerpe T. Strain rate imaging in normal and reduced diastolic function: comparison with pulsed Doppler tissue imaging of the mitral annulus. J Am Soc Echocardiogr. 2001; 14: $264-74$. http://dx.doi.org/10.1067/mje.2001.110375

[5] Smiseth OA, Stoylen A, Ihlen H. Tissue Doppler imaging for the diagnosis of coronary artery disease. Curr Opin Cardiol. 2004; 19: 421-9. PMid:15316447 http://dx.doi.org/10.1097/01.hco.0000135672.79534.e9

[6] Rappaport D., Adam D, Lysyansky P, Reisner S. Assessment of myocardial regional strain and strain rate by tissue tracking in B-mode echocardiograms, Ultrasound in Med.\& Biol. 2006; 32(8): 1181-1192. http://dx.doi.org/10.1016/j.ultrasmedbio.2006.05.005

[7] Amundsen BH, Helle-Valle T, Edvardsen T, Torp H, Crosby J, Lyseggen E, et al. Noninvasive myocardial strain measurement by speckle tracking echocardiography validation against sonomicrometry and tagged magnetic resonance imaging. J Am Coll Cardiol. 2006; 47: 789-793. PMid:16487846 http://dx.doi.org/10.1016/j.jacc.2005.10.040

[8] Cho GY, Chan J, Leano R, Strudwick M, Marwick TH. Comparison of two-dimensional speckle and tissue velocity based strain and validation with harmonic phase magnetic resonance imaging. Am J Cardiol. 2006; 97: 1661-1666. PMid:16728234 http://dx.doi.org/10.1016/j.amjcard.2005.12.063

[9] Goffinet C, Chenot F, Robert A, Pouleur AC, le Polain de Waroux JB, Vancrayenest D, et al. Assessment of subendocardial vs. subepicardial left ventricular rotation and twist using two-dimensional speckle tracking echocardiography: comparison with tagged cardiac magnetic resonance. Eur Heart J. 2009; 30(5): 608-17. PMid:19019994 http://dx.doi.org/10.1093/eurheartj/ehn511

[10] Helle-Valle T, Crosby J, Edvardsen T, Lyseggen E, Amundsen BH, Smith HJ, Rosen BD, et al. New noninvasive method for assessment of left ventricular rotation: speckle tracking echocardiography. Circulation. 2005; 112: 3149-3156. PMid:16286606 http://dx.doi.org/10.1161/CIRCULATIONAHA.104.531558

[11] Notomi Y, Lysyansky P, Setser RM, Shiota T, Popović ZB, Martin-Miklovic MG, et al. Measurement of ventricular torsion by two-dimensional ultrasound speckle tracking imaging. J Am Coll Cardiol. 2005; 45: 2034-2041. PMid:15963406 http://dx.doi.org/10.1016/j.jacc.2005.02.082

[12] Leitman M, Lysyansky P, Sidenko S, Shir V, Peleg E, Binenbaum M, et al. Two-dimensional strain-a novel software for real-time quantitative echocardiographic assessment of myocardial function. J Am Soc Echocardiogr. 2004; 17(10): 1021-9. PMid:15452466 http://dx.doi.org/10.1016/j.echo.2004.06.019

[13] Reisner S, Lysyansky P, Agmon Y, Mutlak D, Lessick J, Friedman Z. Global longitudinal strain: a novel index of left ventricular systolic function. J Am Soc Echocardiogr. 2004; 17: 630-3. PMid:15163933 http://dx.doi.org/10.1016/j.echo.2004.02.011

Published by Sciedu Press 
[14] Liel-Cohen N, Tsadok Y, Beeri R, Lysyansky P, Agmon Y, Feinberg MS, et al. A new tool for automatic assessment of segmental wall motion based on longitudinal $2 \mathrm{~d}$ strain: A multicenter study by the israeli echocardiography research group. Circ Cardiovasc Imaging. 2010; 3: 47-53. PMid:19926829 http://dx.doi.org/10.1161/CIRCIMAGING.108.841874

[15] Bogaert J, and Rademaker FE. Regional nonuniformity of normal adult human left ventricle, Am J Physiol Heart Circ Physiol. 2001; 280: H610-H620. PMid:11158958

[16] Jensen JA and Munk P. Computer phantoms for simulating ultrasound B-mode and CFM images, Acoustical Imaging. 1997; 23: 75-80. Eds.: S. Lees and L. A. Ferrari, Plenum Press.

[17] Jensen JA. Field: A Program for Simulating Ultrasound Systems. Medical \& Biological Engineering \& Computing. 1996; 34: 351-353. PMid:8945858

[18] Jensen JA, Svendsen NB. Calculation of pressure fields from arbitrarily shaped, apodized, and excited ultrasound transducers, IEEE Trans. Ultrason., Ferroelec., Freq. Contr.1992; 39: 262-267.

[19] Park YH, Kang SJ, Song JK, Lee EY, Song JM, Kang DH, et al. Prognostic value of longitudinal strain after primary reperfusion therapy in patients with anterior-wall acute myocardial infarction. J Am Soc Echocardiogr. 2008; 21(3): 262-7. PMid:17904803 http://dx.doi.org/10.1016/j.echo.2007.08.026

[20] Ohara Y, Furuno T, Takahashi T, Hosokawa S, Hiasa Y. Ultrasonic strain versus coronary flow velocity pattern for predicting regional wall motion recovery after primary coronary intervention for acute myocardial infarction. .J Am Soc Echocardiogr. 2008; 21(12): 1369-75. PMid:18930629 http://dx.doi.org/10.1016/j.echo.2008.09.005

[21] Delgado V, Mollema SA, Ypenburg C, Tops LF, van der Wall EE, Schalij MJ, et al. Relation between global left ventricular longitudinal strain assessed with novel automated function imaging and biplane left ventricular ejection fraction in patients with coronary artery disease. J Am Soc Echocardiogr. 2008; 21(11): 1244-50. PMid:18992675 http://dx.doi.org/10.1016/j.echo.2008.08.010

[22] Bland JM, Altman DG. Statistical methods for assessing agreement between two methods of clinical measurement. Lancet. 1986; 1(8476): 307-310. http://dx.doi.org/10.1016/S0140-6736(86)90837-8

[23] Hittinger L, Patrick T, Ihara T, Hasebe N, Shen YT, Kalthof B, et al. Exercise induces cardiac dysfunction in both moderate, compensated and severe hypertrophy. Circulation. 1994; 89; 2219-2231. PMid:8181148

[24] Leitman M, Lysiansky M, Lysyansky P, Friedman Z, Tyomkin V, Fuchs T, et al. Circumferential and longitudinal strain in 3 myocardial layers in normal subjects and in patients with regional left ventricular dysfunction. J Am Soc Echocardiogr. 2010; 23: 64-70. PMid:20122496 http://dx.doi.org/10.1016/j.echo.2009.10.004

[25] Becker M, Ocklenburg C, Altiok E, Futing A, Balzer J, Krombach G, et al. Impact of infarct transmurality on layer-specific impairment of myocardial function: A myocardial deformation imaging study. Eur Heart J. 2009; 30: 1467-1476. PMid:19351689 http://dx.doi.org/10.1093/eurheartj/ehp112

[26] Leitman M, Bachner-Hinenzon N, Adam D, Fuchs T, Teodorovich N, Peleg E, Krakover R, et al. Speckle tracking imaging in acute inflammatory pericardial disease. Echocardiography journal. 2011;28(5):548-55.

[27] Kallel F, Bertrand M, Meunier J. Speckle motion artifact under tissue rotation. IEEE Trans. Ultras. Ferroelec. Freq. Control. 1994; 41(1): 105-122. http://dx.doi.org/10.1109/58.265828

[28] Bachner-Hinenzon N Ertracht O, Lysiansky M, Binah O, Adam D. Layer-specific assessment of left ventricular function by utilizing wavelet de-noising: A validation study. Med Biol Eng Comput. 2011; 49(1): 3-13.

http://dx.doi.org/10.1007/s11517-010-0662-6

[29] Weidemann F, Wacker C, Rauch A, Bauer WR, Bijnens B, Sutherland GR, et al. Sequential changes of myocardial function during acute myocardial infarction, in the early and chronic phase after coronary intervention described by ultrasonic strain rate imaging. J Am Soc Echocardiogr. 2006; 19: 839-47. PMid:16824992 http://dx.doi.org/10.1016/j.echo.2006.01.024

[30] Weigner AW, Allen GJ, and Bing, OHL. Weak and strong myocardium in series: implications for segmental dysfunction. Am J Physiol. 1978; 235(6): H776-83. PMid:736165

[31] Landesberg A, Markhasin VS, Beyar R, Sideman S. Effect of cellular inhomogeneity on cardiac tissue mechanics based on intracellular control mechanisms. Am J Physiol. 1996; 270(3 Pt 2): H1101-H1114. PMid:8780209 